The complexity of multilingual, diglossic or monolingual contexts and their effect on language development are such that they must be analysed with well-adapted tools in order to go beyond traditional analyses based on one or two clearly defined languages.

In terms of social contexts, the Douglas Fir Group (2016) describes micro contexts of social action and interaction, meso contexts of sociocultural institutions and communities and the macro level of ideological structures, which corresponds to what the Council of Europe (2001) also defines.

We should add that a homoglot context is a context where the language to be learnt is spoken, while it is not spoken in a heteroglot environment. Context can also be institutional, guided or unguided, individual or collective. Time (contact time, learning phases, personal history of learning AL) also needs to be analysed as do individual and collective work and interactions.

\title{
Chapter Organisation
}

In Part 1, we show that the focus of our reflection should be both on the interactional dimension of language learning and on the cognitive organisation it enables (Bouchard, 2009). We briefly explore the abovementioned fields in order to highlight the points which influence LLEs and tasks. Our assumption is that those fields and the various theories deal with phenomena that can be viewed as the crystals of a kaleidoscope (see Chapter 4 of Bertin et al., 2010). There are many such crystals and they are studied in so many different fields of research that linking them in a fluid text is not always easy and often artificial. Dealing with them as separate individual crystals is the choice we have made. Each tilt of the kaleidoscope will lead to a different interpretation of how these phenomena can be organised or explained. In the past 10 years, advances in research have modified our conception of some of the crystals. New crystals have been found to be important and new patterns of the crystals are now possible. This part will eventually lead to a checklist of the crystals worth collecting in order to construct appropriate LLEs.

The opening chapters (1-7) in Part 1 present the theoretical underpinnings that precede and influence our choice of case studies in Part 2 and proposed LLEs and tasks in Part 3. They are central to understanding multilingualism. Chapter 1 reviews the interplay between neurophysiology, cognition and language and attempts to uncover the cognitive and neural mechanisms that support language development. Chapter 2 examines this further, but more specifically from a multilingual perspective. We start by examining the relationship between neurophysiology and plurilingualism. The chapter then moves on to consider codeswitching and translanguaging processes of a multilingual's cognition. We draw on recent research to highlight the current situation of plurilingual education and the cognitive advantages of plurilingualism. 
Chapter 3 builds on Chapter 2 by focusing on the shift in multilingual practices and how they are now emphasising holistic approaches that highlight the interrelationship between languages and the benefits of making full use of the plurilingual repertoire. We first examine translation, Content and Language Integrated Learning (CLIL) and multiliteracy approaches. Then, we explore practices from the teacher's perspective outlining the specific competences and training requirements of multilingual teachers.

Chapter 4 revisits useful constructs from psycholinguistics and SLA research that we believe are effective for setting up tasks in a plurilingual approach if the underlying theories are reinterpreted. We describe interactionist, information processing, connectionist and dynamic systems models, as well as the role of attention, mediation and metareflection in language learning.

Chapter 5 attempts to understand the social and cultural dimensions that contribute to multilingual learners' personal development and how this impacts their language development, their identity and actions. We examine the constructs of perception, emotions and motivation and how learners can develop personal agency.

In Chapter 6, we investigate the potential of information and communication technology (ICT) for language learning, highlighting the benefits as revealed by recent research. We describe new learning practices that rely partly on ICT including: informal learning, open educational practices, computer-mediated communication and telecollaboration.

The concluding chapter of Part 1 (Chapter 7) recognises the role of context in understanding language and language learning, but also the complexity associated with trying to analyse context. This leads us to propose a framework of contextual indicators at the macro, meso and micro levels, which are used in our analysis of the various case studies presented in Part 2.

Part 2 (Chapters 8-15) is devoted to a qualitative review and meta-analysis of multilingual research projects in line with our theoretical and practical positions. We bring together 37 case studies from across different contexts, principally in North America, Africa and Europe as the team of authors is more familiar with these areas. The cases do not cover all the multilingual areas in the world and Chapter 14 will show how our findings relate to multilingual settings in other areas, such as Asia and South America. The case studies are purposely varied by age group, educational setting, learning approaches and scale, as well as sociocultural and international contexts. We describe our methods in Chapter 8 and provide a useful summary of the case studies to guide the reader. The case studies in Chapters 9-12 are organised according to their geographical location as mentioned above. Within this overall organisation, we also examine CLIL, translanguaging and intercomprehension learning programmes, as well as very specific studies on just one 
aspect of language development. In addition, Chapter 13 looks specifically at telecollaboration projects. After offering a synthetic overview of what the case studies reveal and shedding light on data from studies of the situation in Asia and South America (Chapter 14), we proceed to propose in Chapter 15 a checklist of the factors to be taken into account when organising LLEs and designing tasks. These factors are the crystals we described previously.

Part 3 aims to bridge the gap between theory and practice as we consider the implications of the case studies and of our theoretical foundations and propose a flexible and adaptive framework for setting up learning environments and designing tasks. The framework takes into account the crystals and the effects of contextual, cultural and/or individual variations. Chapter 16 takes a look at innovative ICT-based multilingual LLEs and provides an extensive list of essential tools, systems and tasks. Chapter 17 highlights aspects of our flexible approach including how teachers and learners can collaborate to co-construct tasks and learning environments and how variability and the sociocultural needs of learners can be taken into account in the designing of courses and tasks in a multilingual perspective. The closing chapter provides a model for this flexible, task-based framework, describing teacher/learner roles and interactions with context, culture, technology and tasks, as well as the relations between all these poles and the dynamic nature of the system. It also examines the way macro/social tasks and micro/training tasks can be designed and implemented. 